\title{
Erratum
}

\section{Investigation of slot-pole combinations on IPM BLDC motor considering magnetization direction}

Hong-seok Kim, Jung-woo Kwon and Byung-il Kwon*

Department of Electronic Systems Engineering, Hanyang University, Ansan, Gyeonggi-do, Korea

[International Journal of Applied Electromagnetics and Mechanics, 59(3) 2019, 865-872, DOI 10.3233/JAE-171126]

https://content.iospress.com/articles/international-journal-of-applied-electromagnetics-andmechanics/jae171126

On page 865 in the printed version of the article, the following author is given: "Byung-il Kwon" However, the full list of authors should be: "Hong-seok Kim*, Jung-woo Kwon*, Byung-il Kwon*

*Department of Electronic Systems Engineering, Hanyang University, Ansan, Gyeonggi-do, Korea"

The online version of the article has been corrected.

\footnotetext{
*Corresponding author: Byung-il Kwon, Department of Electronic Systems Engineering, Hanyang University, Ansan, Gyeonggi-do, Korea. E-mail: bikwon@hanyang.ac.kr.
} 